\title{
A bias generating temporal distortions in serial perception
}

\author{
Franklenin Sierra, ${ }^{1}$ David Poeppel,,${ }^{1,2}$ and Alessandro Tavano ${ }^{1}$
}

$4{ }^{1}$ Department of Neuroscience, Max Planck Institute for Empirical Aesthetics (Frankfurt am Main,

5 Hesse, Germany)

$6 \quad{ }^{2}$ Department of Psychology, New York University (New York City, NY, USA)

8 Corresponding author: Franklenin Sierra < franklenin.sierra@protonmail.com>.

9 Department of Neuroscience, Max Planck Institute for Empirical Aesthetics.

10 Grüneburgweg 14, 60322 Frankfurt am Main, Germany.

\section{Summary}

13 A precise estimation of event timing is essential for survival. ${ }^{1}$ Yet, temporal distortions are ubiquitous

14 in our daily sensory experience. ${ }^{2}$ A specific type of temporal distortion is the time order error (TOE),

15 which occurs when estimating the duration of events organized in a series. ${ }^{3}$ TOEs shrink or dilate

16 objective event duration. Understanding the mechanics of subjective time distortions is fundamental

17 since we perceive events in a series, not in isolation. In previous work, ${ }^{4}$ we showed that TOEs appear

18 when discriminating small duration differences $(20$ or $60 \mathrm{~ms}$ ) between two short events (Standard, S

19 and Comparison, C), but only if the interval between events is shorter than 1 second. TOEs have been

20 variously attributed to sensory desensitization, ${ }^{5,6}$ reduced temporal attention, ${ }^{7,4}$ poor sensory weighting 21 of $\mathrm{C}$ relative to $\mathrm{S},{ }^{8}$ or idiosyncratic response bias. ${ }^{6}$

22 Surprisingly, the serial dynamics of relative event duration were never considered as a factor

23 generating TOEs. In two experiments we tested them by swapping the order of presentation of $S$ and

24 C. Bayesian hierarchical modelling showed that TOEs emerge when the first event in a series is shorter

25 than the second event, independently of event type ( $\mathrm{S}$ or $\mathrm{C}$ ), sensory precision or individual response 
26 bias. Participants disproportionately expanded first-position shorter events. Significantly fewer errors

27 were made when the first event was objectively longer, confirming the inference of a strong bias in

28 perceiving ordered event durations. Our finding identifies a hitherto unknown duration-dependent

29 encoding inefficiency in human serial perception.

\section{Results}

32 In two separate experiments, we asked human participants to discriminate the duration of a Standard

33 (S) event against that of a Comparison (C) event (or vice versa), and decide which one was longer 34 (two-interval forced choice design, 2IFC). The S event was displayed in first position in Experiment

35 1, but was shifted to second position in Experiment 2 (Fig 1a). To signal the onset and offset of each 36 event, we used a blue disk as a visual stimulus (see STAR Methods). The interval between cue and 37 first stimulus onset was randomly chosen between 400 and $800 \mathrm{~ms}$. The duration of the S event (120

$38 \mathrm{~ms}$ ) was kept constant, while the duration of the $C$ event varied, providing participants with three 39 degrees of sensory evidence (weak $\pm \Delta 20$; medium $\pm \Delta 60$; strong $\pm \Delta 100$; Fig $1 b$ ). Temporal 40 attention was manipulated by parametrically increasing the Inter-Stimulus Interval (ISI) between $\mathrm{S}$ and 41 C (or C and S): 400, 800, 1600, and $2000 \mathrm{~ms}$.

42 We hypothesized that with an ever-changing $\mathrm{C}$ in first position (second experiment) the 43 magnitude of time order error (TOE) would increase, as participants would not benefit from orienting 44 attention in time to $\mathrm{C}$ in the second position (first experiment). Hence, we expected an interaction 45 between the factors Stimulus presentation order and the orienting of attention in time. ${ }^{9}$ 
a)

\section{Experiment 1}

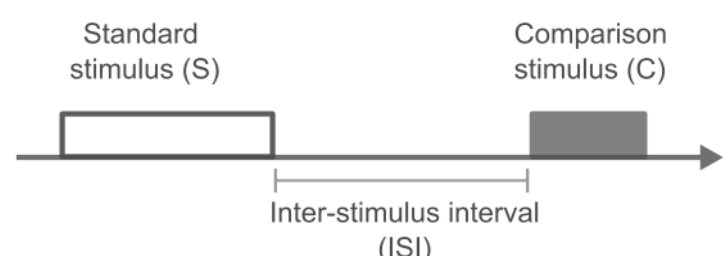

(ISI)

\section{Experiment 2}

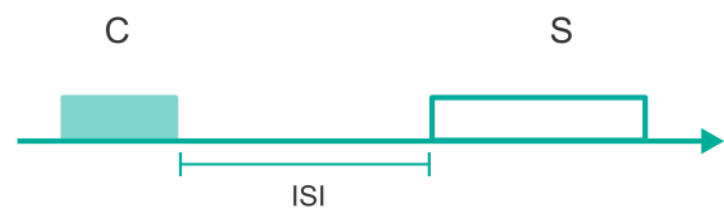

c)

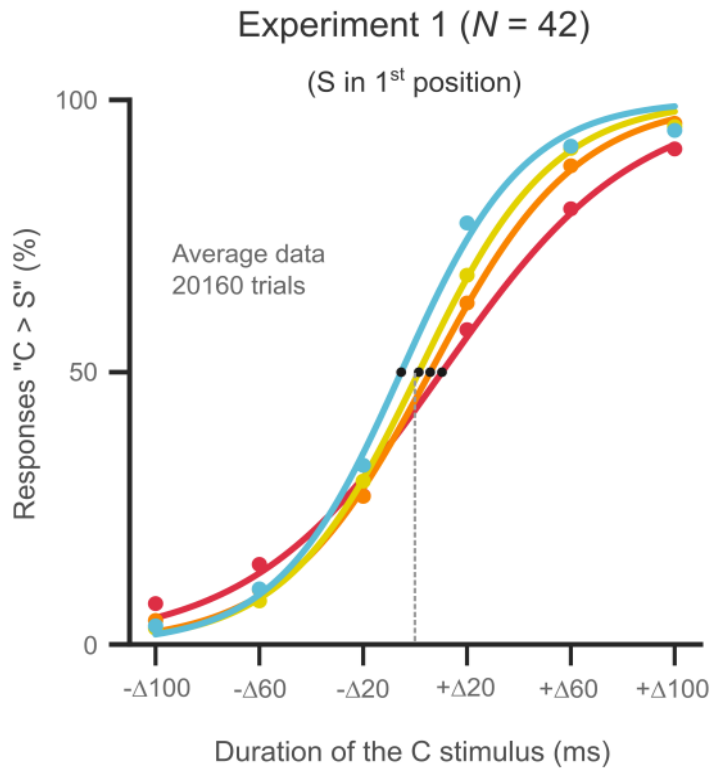

b)

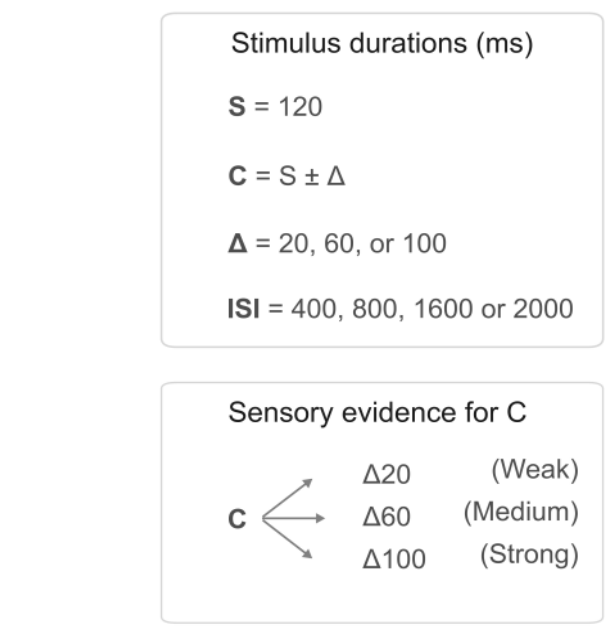

Experiment $2(N=44)$

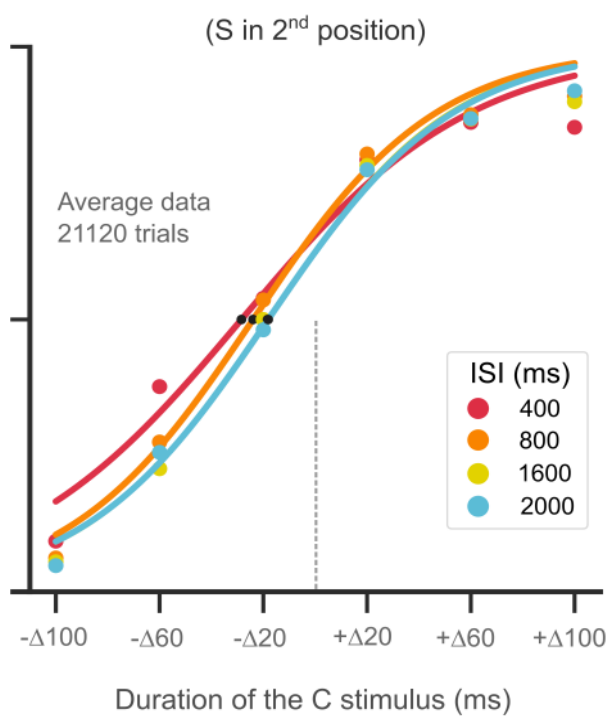

48 Fig. 1. Two-interval forced choice (2IFC) task and model of responses. (a) Timeline of events in the

49 2IFC task. The Standard stimulus (S) was displayed in the $1^{\text {st }}$ position (experiment 1 ), and in the $2^{\text {nd }}$

50 position (experiment 2). We implemented four Inter-stimulus intervals (ISIs) levels: 400, 800, 1600

51 and $2000 \mathrm{~ms}$. (b) The Standard stimulus had a fixed duration of $120 \mathrm{~ms}$, whereas the durations for

52 the Comparison stimulus varied according to its level of sensory evidence: Weak, Medium, and

53 Strong. (c) Psychometric curves modeling performance on each ISI level of experiments 1 and 2.

54 Points represent data pooled across participants. Lines depict separate fits for each ISI condition, the 
55 gray vertical line depict the physical magnitude $\phi_{s}$ of the Standard stimulus, black dots represent the 56 point of subjective equality (PSE) for each ISI condition. The just noticeable difference (JND) is

57 computed from the slopes $\beta$ of the sigmoid curves. Contrary to experiment 1 , all PSEs are shifted to

58 the left side in experiment 2, which exemplifies the time order effect.

\section{Sensory precision and temporal distortion profiles}

61 Responses were modeled using individual sigmoid psychometric functions for each ISI level, from 62 which we obtained the midpoint $\mu$ and the slope $\beta$ of each curve (Fig 1c). After fitting the responses, 63 we determined the point of subjective equality (PSE) between S and C, which corresponds to the 64 magnitude $\mu$. We analyzed precision indexed by the just noticeable difference (JND), and the 65 cumulative effect time order errors (TOEs) indexed by the constant error (CE). CE is a global index

66 of temporal distortion. We computed the JND as the $\beta$ multiplied by the $\log \left(\frac{0.75}{0.25}\right),{ }^{10,5}$ and the CE as 67 the difference between the physical magnitude $\phi_{s}$ of the Standard event and $\mu$.

To test changes in the dependent variables as a function of factors ISI and Stimulus presentation order ( $\mathrm{S}$ first or $\mathrm{C}$ first), we quantified the effect of both factors by implementing Bayesian Model Comparison. To do that, we applied a Bayes factor approach to a mixed ANOVA using Bayes' rule. ${ }^{11,12,13}$ We built four alternative models: the $M_{\text {ISI }}$ model (using the ISI as

72 predictor), the $M_{\mathrm{E}}$ model (using Experiment as predictor, that is Standard position), the $M_{\mathrm{ISI}+\mathrm{E}}$ (using

73 both factors as predictors), and the $M_{\text {Int }}$ model (including ISI and Experiment predictors, but also their

74 interaction). To evaluate the predictive performance of each model, we compared them to the null

75 model $M_{0}$ (no effect of ISI and Stimulus presentation order) by computing the Bayes factor. ${ }^{9}$

Just Noticeable Difference (JND). In experiment 1, the mean JND values were: ISI $_{400}=$

$29.90(S D=15.91), \mathrm{ISI}_{800}=22.54(S D=9.59), \mathrm{ISI}_{1600}=22.11(S D=10.54)$, and $\mathrm{ISI}_{2000}=20.98(S D$

$78=11.54)$. In experiment 2 , they were $32.67(S D=17.05), 23.97(S D=12.01), 25.53(S D=14.52)$, and

$7926.96(S D=14.62)$, for the $\mathrm{ISI}_{400}, \mathrm{IS}_{1800}, \mathrm{ISI}_{1600}$ and $\mathrm{ISI}_{2000}$ conditions, respectively (Fig $\left.\mathbf{2 a}\right)$.

80 Modeling of the JND data revealed that the data were best explained by the $M_{\mathrm{ISI}}$ model $\left(\mathrm{BF}_{10}=\right.$ 81 107822; see Fig 2b). Post hoc comparisons revealed decisive evidence in favor of statistical 82 differences between the ISI $_{400}$ and the remaining conditions: ISI $_{800}$, ISI $_{1600}$, and ISI $_{2000}$ (posterior odds $83=53596,284$, and 109; respectively). We conclude that Standard position (Experiment factor) does

84 not have modulatory effects on sensory precision, which is driven by temporal attention. 
85 Constant error (CE). In experiment 1, mean CE values decreased with increasing ISI: ISI $_{400}=$ $869.18(S D=19.32), \operatorname{ISI}_{800}=7.18(S D=14.50), \mathrm{ISI}_{1600}=1.94(S D=12.34)$, and $\mathrm{ISI}_{2000}=-2.60(S D=$ 87 11.30). We found a similar pattern in experiment 2: $\operatorname{ISI}_{400}(M=27.97, S D=22.82), \operatorname{ISI}_{800}(M=$ 88 24.02, $S D=20.12), I_{1} I_{1600}(M=20.62, S D=20.79)$, and $I_{S I} I_{2000}(M=18.26, S D=20.78 ;$ Fig $2 c)$.

89 These results were best explained by the $M_{\mathrm{ISI}+\mathrm{E}}$ model $\left(\mathrm{BF}_{10}=185.6 * 10^{10}\right)$. Post hoc 90 comparisons revealed very strong evidence in favor of differences between the ISI $_{400}$ and the 91 conditions $\mathrm{ISI}_{1600}$ and $\mathrm{ISI}_{2000}$ (posterior odds $=56,56$ and 3189; respectively), but also between 92 the $\mathrm{ISI}_{800}$ and the conditions $\mathrm{ISI}_{1600}$ and $\mathrm{ISI}_{2000}$ conditions (posterior odds $=6$ and 118; respectively).

93 The Experiment factor revealed decisive evidence in favor of a statistical difference between 94 experiments 1 and 2 (posterior odds $=4.1 * 10^{15}$ ). That is, increasing ISI helped to minimize CE in 95 both experiments. Participants in experiment 2 made more errors than participants in experiment 1 96 (Fig 2d). 
a)

\section{Experiment 1}

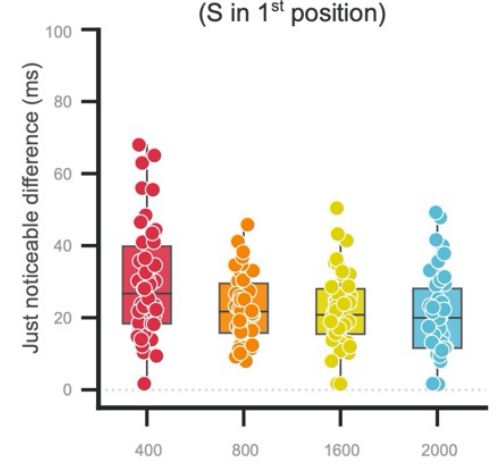

c)

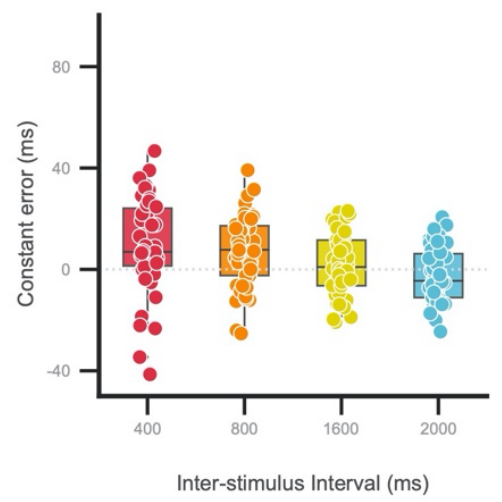

Experiment 2
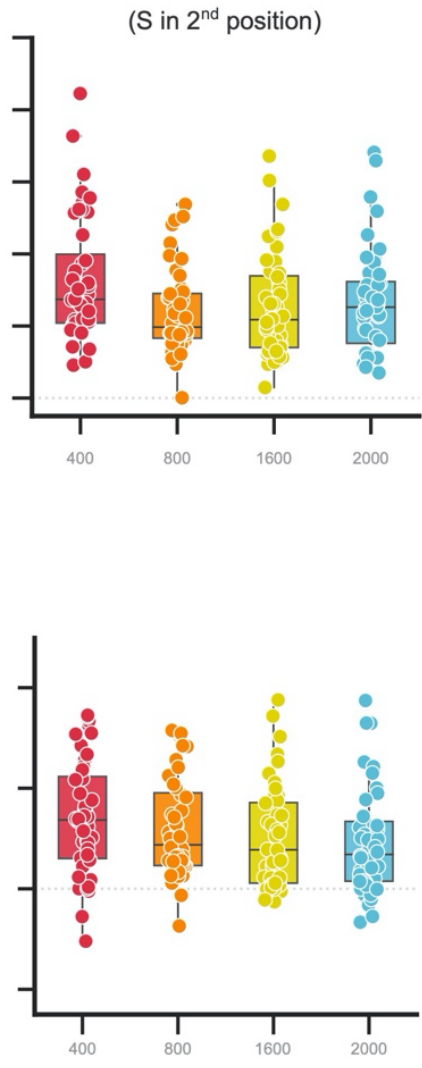

Inter-stimulus Interval (ms) b) Model-Averaged Posterior Distributions

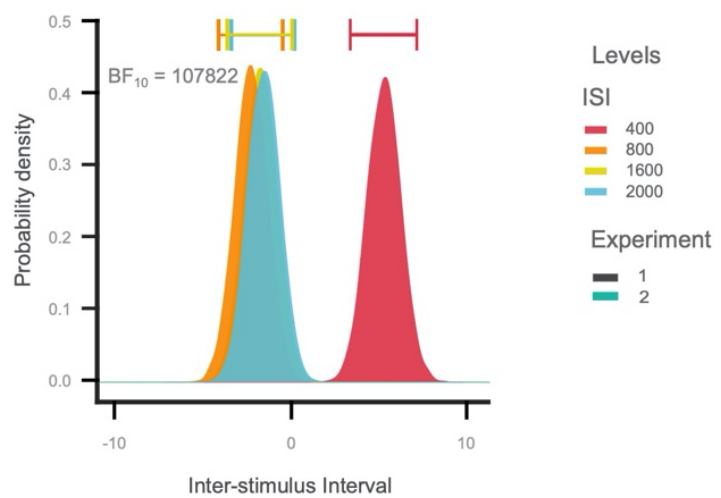

d)

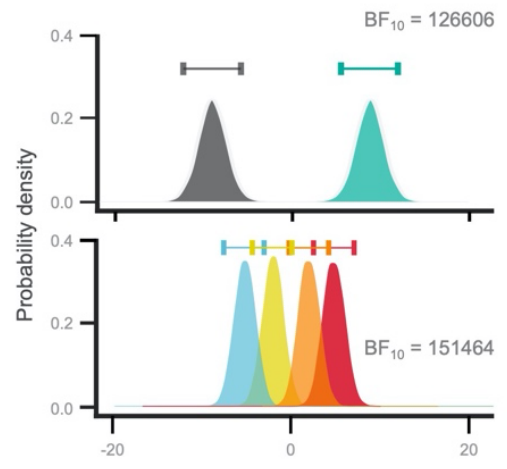

Inter-stimulus Interval

97 Fig. 2. Sensory precision, temporal distortions and model-averaged posterior distributions of the

98 Bayesian models. (a) Just noticeable difference (JND) data plotted as a function of ISI. Box-and-

99 whisker plots show the distribution of the JND. The bottom and top edges indicate the interquartile

100 range (IQR), whereas the median is represented by the horizontal line. The extension of the whiskers

101 is 1.5 times the IQR. Boxplots are overlaid with data of each participant. Results from both

102 experiments seem to follow the same pattern. (b) Bayesian Model Comparison revealed that the JND

103 data were best explained by the $\mathrm{M}_{\mathrm{ISI}}$ model, indicating that, the position of the Standard stimulus does

104 not have modulatory effects on the temporal sensitivity. This result can be visually verified by the clear

105 separation between the distribution ISI $_{400}$ and the rest of the posterior distributions. (c) Constant

106 errors (CEs) plotted as a function of ISI. We can observe higher and positive CEs values for

107 experiment 2. (d) Distributions of the $\mathrm{M}_{\mathrm{ISI}}$ model signal an effect of ISI, whereas the separated

108 distributions on the $\mathrm{M}_{\mathrm{E}}$ model reveal an effect of Experiment (i.e., Standard position). Consequently, 
109 the Bayes factor revealed that CE data were best modeled by the inferential model including both

110 factors, that is the $\mathrm{M}_{\mathrm{ISI}+\mathrm{E}}$ model.

111

112 Relative duration effects

113 We then analyzed individual discrimination performance at each sensory evidence level

$114( \pm \Delta 100, \pm \Delta 60, \pm \Delta 20)$. We tested how the percentage of accuracy changes as a function of relative

115 duration of the stimuli within a series: "First stimulus longer than the second" and "Second stimulus

116 longer than the first" (Fig 3a,c,e). For each sensory evidence level and duration ordering, we again

117 applied the four Bayesian inferential models ( $M_{\mathrm{ISI}}, M_{\mathrm{E}}, M_{\mathrm{ISI}+\mathrm{E}}$, and $\left.M_{\mathrm{Int}}\right)$ and compared them to the null

118 model $M_{0}$. These models were carried out after applying an arcsine transformation to the data. This is

119 calculated as two times the arcsine of the square root of the proportion of correct responses.

120 Strong sensory evidence $( \pm \Delta 100)$. When the first stimulus was longer, mean accuracy

121 values in experiment 1 were: ISI $_{400}=92.50$, ISI $_{800}=96.42$, ISI $_{1600}=97.38$, and ISI $_{2000}=96.54$. In

122 experiment 2 , they were $93.75,96.59,96.70$, and 97.50, respectively. When the second stimulus was

123 longer, mean accuracy values in experiment 1 were: $\mathrm{ISI}_{400}=94.40, \mathrm{ISI}_{800}=97.02, \mathrm{ISI}_{1600}=97.26$, and

$124 \mathrm{ISI}_{2000}=95.83$. Whereas in experiment 2 , they were 90.79, 96.13, 96.02, and 96.02, respectively.

125 The best model was the $M_{\text {ISI }}$ model independently of the serial position of the longer stimulus

$126\left(\mathrm{BF}_{10}=11895.5 ; \mathrm{BF}_{10}=13.5\right.$; respectively; Fig $\left.3 \mathbf{b}\right)$. That is, when the sensory evidence for a difference

127 between $\mathrm{S}$ and $\mathrm{C}$ is strong, behavioral performance is explained solely by the ISI factor, and the relative

128 duration of events in a series is weighted out. When the first stimulus was longer, post hoc

129 comparisons revealed very strong top decisive evidence in favor of a difference between the ISI 400 and

130 the remaining conditions (ISI ${ }_{800}, \mathrm{ISI}_{1600}$, and $\mathrm{ISI}_{2000}$, posterior odds $=155,474$, and 88; respectively).

131 Whereas when the second stimulus was longer, we found moderate evidence in favor of a difference

132 between the ISI $_{400}$ and the long ISI conditions $\left(\mathrm{ISI}_{1600}\right.$ and $\mathrm{ISI}_{2000}$, posterior odds $=4$ and 3 ;

133 respectively). We found no evidence for a difference between ISI $_{400}$ and ISI $_{800}$ (posteriors odds $=1$ ).

134 Medium sensory evidence $( \pm \Delta 60)$. When the first stimulus was longer, mean accuracy

135 values in experiment 1 were: $\mathrm{ISI}_{400}=89.28, \mathrm{ISI}_{800}=94.16, \mathrm{ISI}_{1600}=95.47$, and $\mathrm{ISI}_{2000}=93.69$. Whereas

136 in experiment 2 , they were $68.75,78.63,81.36$, and 80.00 , respectively. When the second stimulus was

137 longer, mean accuracy values in experiment 1 were: $\mathrm{ISI}_{400}=85.23, \mathrm{ISI}_{800}=91.54, \mathrm{ISI}_{1600}=93.09$, and

$138 \mathrm{ISI}_{2000}=92.73$. In experiment 2 , they were 92.27, 93.29, 91.47, and 92.04, respectively. 
When the first stimulus was effectively longer, the accuracy data were best explained under

140 the Null model $\left(M_{\mathrm{E}}, \mathrm{BF}_{10}=0.31 ; M_{\mathrm{ISI}}, \mathrm{BF}_{10}=0.15 ; \mathrm{M}_{\mathrm{Int}}, \mathrm{BF}_{10}=0.12 ; M_{\mathrm{E}+\mathrm{ISI}}, \mathrm{BF}_{10}=0.04\right)$. When the

141 second stimulus was longer, the $M_{\mathrm{E}+\mathrm{ISI}}$ was the best model $\left(\mathrm{BF}_{10}=1.66 * 10^{11}\right.$; Fig $\left.\mathbf{3 d}\right)$. Post hoc

142 comparisons of the ISI factor revealed that there was decisive evidence for differences between the

$143 \mathrm{ISI}_{400}$ and the rest of the ISI levels $\left(\mathrm{ISI}_{800}, \mathrm{ISI}_{1600}, \mathrm{ISI}_{2000}\right.$, posterior odds $=35985,2.7 * 10^{6}$, and 36531;

144 respectively). The Experiment factor revealed too that there is decisive evidence for a difference

145 between experiments 1 and 2 (posterior odds $=2.3 * 10^{7}$ ).

146 Weak sensory evidence $( \pm \Delta 20)$. When the first stimulus was longer, mean accuracy values

147 in experiment 1 were: $\mathrm{ISI}_{400}=75.83, \mathrm{ISI}_{800}=78.45, \mathrm{ISI}_{1600}=72.38$, and $\mathrm{ISI}_{2000}=71.90$. Whereas in

148 experiment 2 , they were 47.5, 49.77, 52.38, and 55.45, respectively. When the second stimulus was

149 longer, mean accuracy values in experiment 1 were: ISI $_{400}=59.52$, ISI $_{800}=63.92$, ISI $_{1600}=69.04$, and

$150 \mathrm{ISI}_{2000}=78.33$. In experiment 2 , they were 83.06, 85.68, 82.50, and 80.90, respectively.

151 When first stimulus was longer, the data were best explained by the $M_{\mathrm{E}+\mathrm{ISI}}$ model $\left(\mathrm{BF}_{10}=\right.$

152 261.9). Post hoc comparisons of the ISI factor revealed moderate evidence for differences between

153 the ISI $_{800}$ and the ISI levels 16000 and 2000 (posteriors odds $=7$ and 10, respectively). We found no

154 differences between the rest of the conditions (all prior odds $\leqq 0.3$ ). The Experiment factor revealed

155 decisive evidence for a difference between experiments 1 and 2 (posteriors odds $=35586$ ).

156 When the longer stimulus was in the second position, the data were best explained by

157 the $M_{\text {Int }}$ model $\left(\mathrm{BF}_{10}=9.5 * 10^{9}\right.$; Fig 3f $)$. Post-hoc analyses of the ISI factor revealed that there are

158 moderate and decisive evidence for differences between the ISI $_{400}$ and the conditions ISI $_{1600}$ and ISI $_{2000}$

159 (posteriors odds $=3$ and 8824, respectively), but also decisive and moderate evidence for differences

160 between the conditions ISI $_{2000}$ when compared to the conditions ISI $_{800}$ and ISI1 $_{600}$ (posteriors odds $=$

1612137 and 10, respectively). The Experiment factor revealed decisive evidence in favor of a difference

162 between experiments 1 and 2 (posteriors odds $=4 * 10^{9}$ ).

163 In experiment 1, post hoc comparisons of the interaction revealed differences between the

$164 \mathrm{ISI}_{400}$ and the conditions $\mathrm{ISI}_{1600}$ and $\mathrm{ISI}_{2000}$ (posteriors odds $=8$ and 50889, respectively), but also

165 between the conditions ISI $_{2000}$ when compared to the conditions ISI $_{800}$ and ISI $_{1600}$ (posteriors odds $=$

1661240 and 29, respectively). In Experiment 2, post hoc comparisons revealed that data were best

167 explained under the Null model (all posterior odds $\leqq 0.43$ ). 
a)

Strong sensory evidence $( \pm \Delta 100)$

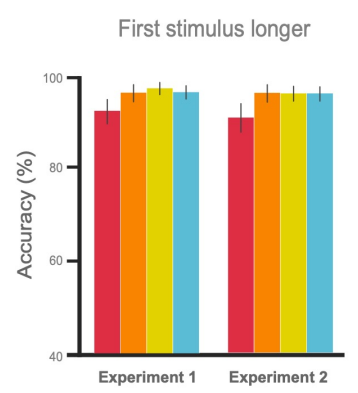

Second stimulus longer

c)

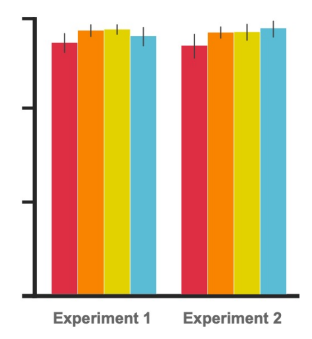

Medium sensory evidence $( \pm \Delta 60)$

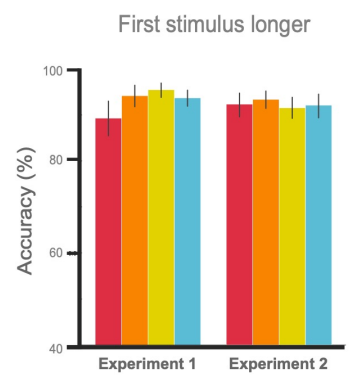

Second stimulus longer

e)
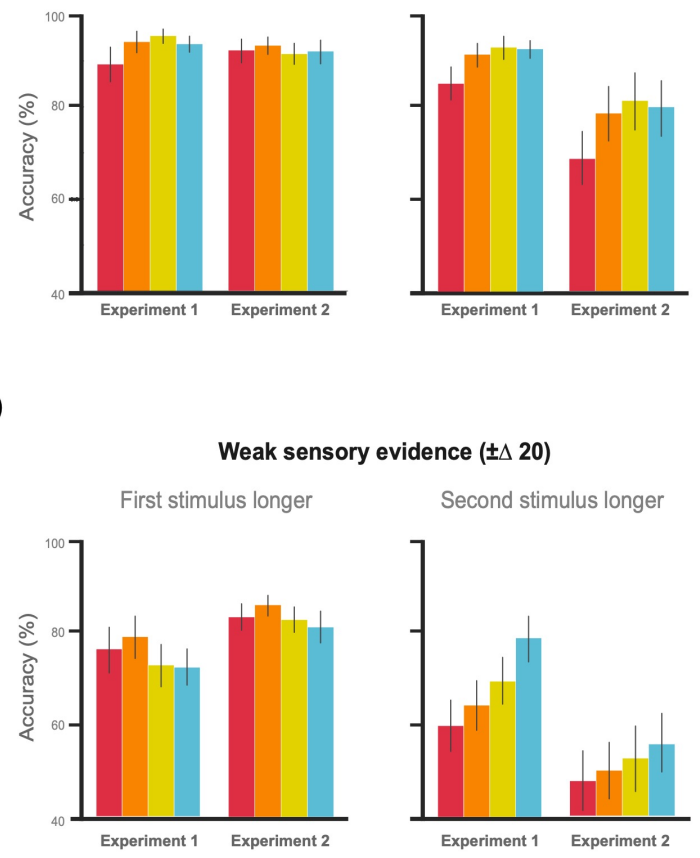

b)

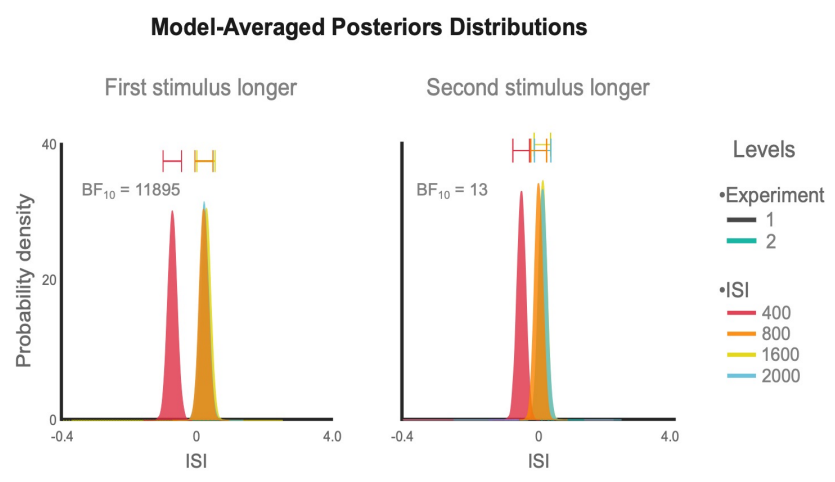

d)

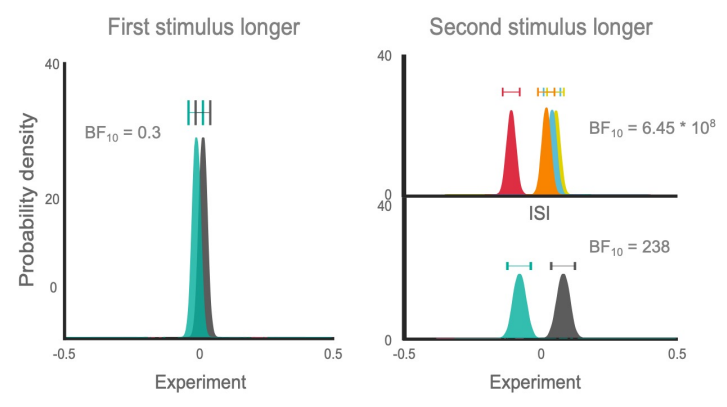

f)

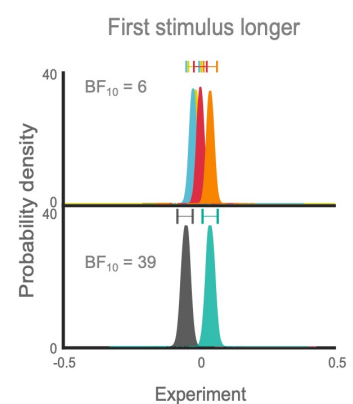

Second stimulus longer

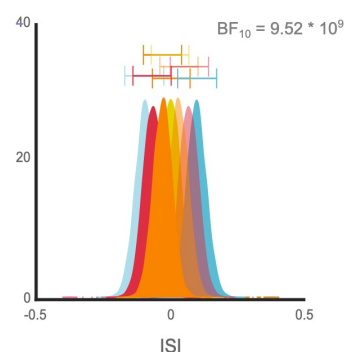

169 Fig. 3. Accuracy and model-averaged posterior distributions of the Bayesian models. a) Strong sensory

170 evidence. Accuracy divided by the position of the longer stimulus, and plotted as a function of ISI and

171 Experiment. Results follow the same pattern independently of the experiment. b) Posterior

172 distributions of the MISI model confirmed that differences between experimental conditions - in the

173 strong sensory evidence - are elicited by the ISI factor. c) For the Medium sensory evidence, when

174 the first stimulus was longer, we found no differences between Experiments or ISI levels. However,

175 the opposite occurred when the second stimulus was longer: we observed a main effect of both 
176 factors. d) Posteriors distribution of the medium evidence category: the model $M_{\mathrm{E}}$ fails to explain the

177 data for the conditions with first stimulus longer, but for the opposite conditions the data were best 178 modeled by the inferential model including both factors, that is the Model $M_{\mathrm{E}+\mathrm{ISI}}$. e) In the Weak 179 sensory category, we observed larger differences in accuracy between experiments and ISI 180 levels. f) Posterior distributions show that for first and second stimulus longer the best models 181 were the $M_{\mathrm{E}+\mathrm{ISI}}$ and the $M_{\mathrm{Int}}$ models, respectively. (Lighter colors signal distributions of the 182 experiment 2).

\section{A bias in serial duration perception}

185 We then used a Bayesian hierarchical model to compare differences in the accuracy rate between "First 186 stimulus longer than the second" $\left(\phi^{a}\right)$ and "Second stimulus longer than the first" $\left(\phi^{b}\right)$ conditions

187 for the uncertain sensory evidence levels $\pm \Delta 20$ and $\pm \Delta 60$, which showed an effect of the 188 experimental manipulation (for the Strong sensory evidence, see Supplemental Information). To do 189 that, we applied the Bayesian rate comparison model ${ }^{14}$ and quantified the difference $\alpha$ between both 190 rates $\left(\phi^{a}\right.$ and $\left.\phi^{b}\right)$ as a normally distributed random effect (see STAR Methods; Fig. 4a).

We applied this model in both experiments for within comparisons at each ISI level. Thus, at each ISI level of each experiment we compared the null hypothesis $H_{0}$ (no difference in accuracy

193 between $\phi^{a}$ and $\phi^{b}$ rates) versus the alternative hypothesis $H_{1}$ (Fig. 4b).

194 Medium sensory evidence $( \pm \Delta 60)$. Results of experiment 1 yielded strong and moderate 195 evidence in favor of $H_{1}$ in the conditions $\mathrm{ISI}_{400}$ and $\mathrm{ISI}_{800}\left(\mathrm{BF}_{10}=12 ; \mathrm{BF}_{10}=9\right.$; respectively), but no 196 evidence in the longer conditions $\mathrm{ISI}_{1600}$ and $\mathrm{ISI}_{2000}\left(\mathrm{BF}_{10}=3 ; \mathrm{BF}_{10}=0.86\right.$; anecdotal evidence in favor 197 of $H_{1}$ and $H_{0}$, respectively; Fig 4c). These results confirm that, for short ISIs, participants do more 198 mistakes when the longer stimulus is place in the second position. However, this effect can be 199 minimized by the allocation of attention in time. ${ }^{7,15}$

200 Contrary to experiment 1, results of experiment 2 showed decisive evidence for $H_{1}$ at all ISI 201 levels: $\mathrm{ISI}_{400}, \mathrm{ISI}_{800}, \mathrm{ISI}_{1600}$ and $\mathrm{ISI}_{2000}\left(\mathrm{BF}_{10}>10000 ; \mathrm{BF}_{10}>10000 ; \mathrm{BF}_{10}=310 ; \mathrm{BF}_{10}=1512\right.$; 202 respectively; Fig 4d). That is, when sensory evidence is of medium strength, temporal attentions does 203 not help to minimize temporal errors. Regardless of the ISI, participants do more mistakes when the 204 longer stimulus is placed in the second position. 
205 Weak sensory evidence $( \pm \Delta 20)$. We found similar results in the weak sensory evidence. In

206 experiment 1, results yielded extreme evidence in favor of $H_{1}$ in the conditions $\mathrm{ISI}_{400}$ and $\mathrm{ISI}_{800}\left(\mathrm{BF}_{10}\right.$

$207=2945 ; \mathrm{BF}_{10}=3013$; respectively $)$, but no differences in the longer ISIs: 1600 and $2000\left(\mathrm{BF}_{10}=0.59\right.$;

$208 \mathrm{BF}_{10}=2$; respectively; Fig. 4e). In experiment 2, results showed extreme evidence for $H_{1}$ at each ISI

209 level $\left(\mathrm{BF}_{10}>10000 ; \mathrm{BF}_{10}>10000 ; \mathrm{BF}_{10}>10000 ; \mathrm{BF}_{10}>10000\right.$; respectively; Fig. 4f). 
a)

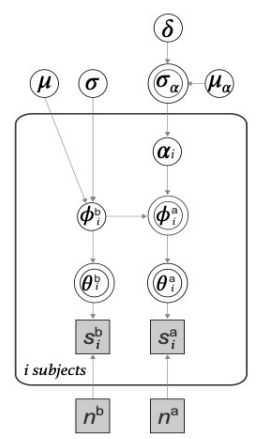

c)

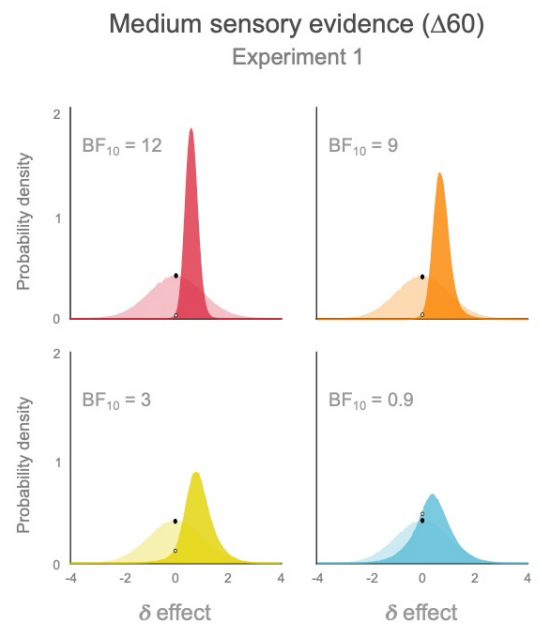

e)

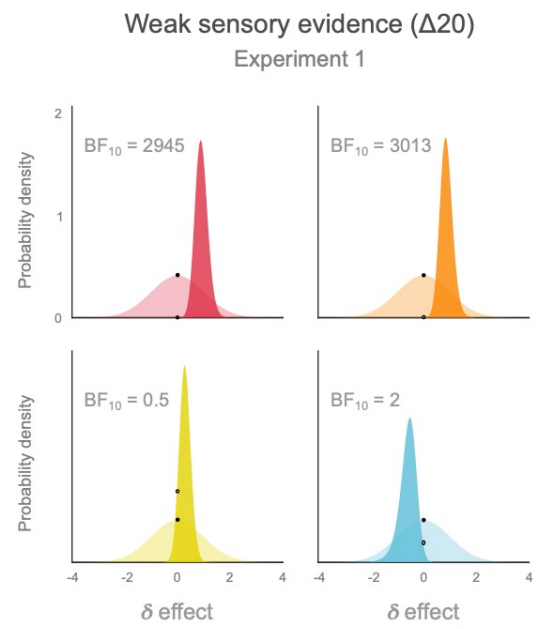

b)

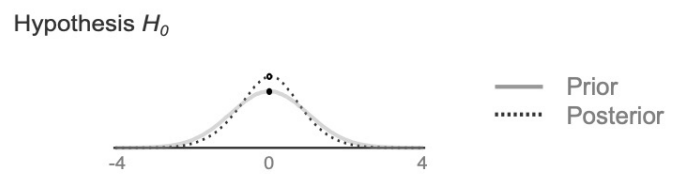

Hypothesis $H_{1}$

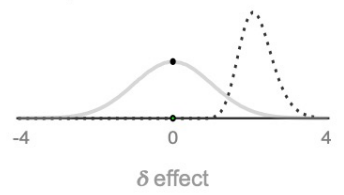

d)

Medium sensory evidence $(\Delta 60)$ Experiment 2
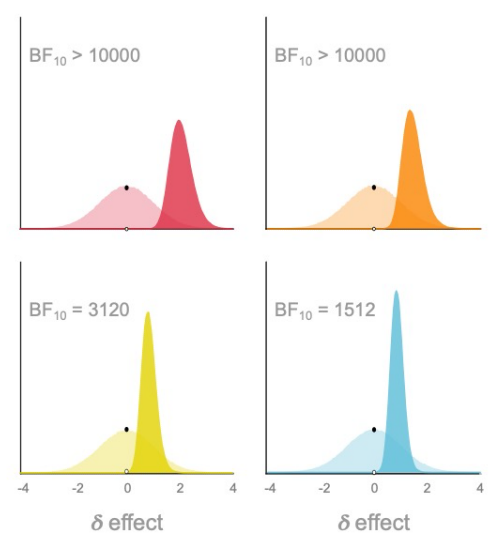

ISI Levels

Prior Posterior

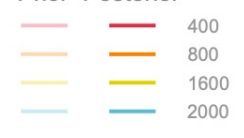

f)

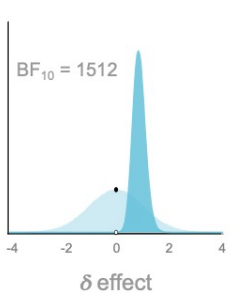

Weak sensory evidence $(\Delta 20)$ Experiment 2

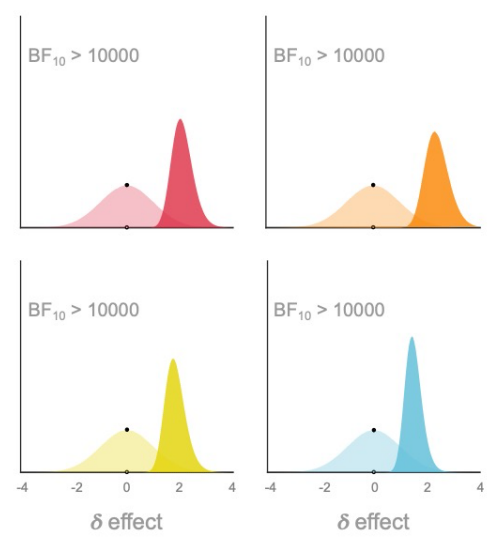

210 Fig. 4. Bayesian rate-comparison model, hypotheses, and prior and posteriors distributions. a) 211 Graphical illustration of the Bayesian rate-comparison model. We tested whether differences $\alpha$ in the 
212 accuracy rates $\phi^{a}$ and $\phi^{b}$ are dependent on having the longer stimulus in the first position. To

213 quantify the effect size, we used the parameter $\delta$. b) Our Bayesian model compared the alternative

214 hypothesis $\mathrm{H}_{1}$ (differences in accuracy rate between the conditions "First stimulus longer" and

215 "Second stimulus longer") versus the null hypothesis $\mathrm{H}_{0}$ (no differences between conditions).

216 Overlapping distributions signal evidence for $\mathrm{H}_{0}$, whereas a shift of the posterior distribution signals

217 evidence in favor of $\mathrm{H}_{1}$. c) For the Medium sensory evidence, we found that posteriors distributions

218 -in experiment 1 - yielded evidence in favor of $\mathrm{H}_{1}$ for the short ISIs conditions, but no evidence in

219 the long ISIs. That is, longer waiting times between stimuli lead to a better temporal discrimination.

220 However, this does not occur when the ISI is short, as we can see as we can observe differences in

221 behavioral performance between the conditions "First stimulus longer" longer" and "Second stimulus

222 longer". d) For experiment 2, all posterior distributions yielded extreme evidence in favor of $\mathrm{H}_{1}$. e-f)

223 We found the same pattern of results for both experiments in the Weak sensory evidence category: In

224 experiment 1 , short ISIs (400 and $800 \mathrm{~ms}$ ) yielded evidence for $\mathrm{H}_{1}$, but we found no evidence in favor

225 in the long ISIs. In experiment 2, all posterior distribution yielded evidence in favor of $\mathrm{H}_{1}$. Overall,

226 these results show that by placing the longer stimulus in the first position, temporal accuracy can be

227 increased.

\section{Response bias}

230 Finally, we tested whether participants were biased in hitting the keys for "S" or "C". We abstracted

231 away from the confounding factor of sensory uncertainty by analyzing key responses across ISI levels

232 at the Strong sensory evidence level only $( \pm \Delta 100)$. We applied individual Bayesian binomial analyses

233 to the set of responses, and verifed the null hypothesis $H_{0}$ of a $50 \%$ probability to choose either $\mathrm{S}$ or

234 C. In both experiments, results yielded Bayes factors $\left(\mathrm{BF}_{01}\right)$ with moderate evidence in favor of the

235 null hypothesis (Supplemental Information Fig 2). For experiment 1, the lower and higher values

236 for the BF were 4.5 and 10.1, respectively. Whereas for experiment 2, they were 3.6 and 10.1. Overall,

237 button press data were between 3.6 and 10.1 more likely under the null hypothesis (no bias) than under

238 the alternative hypothesis, discarding the presence of an idiosyncratic response bias in some of the 239 participants. 


\section{Discussion}

242 Time order error (TOE) is a subjective distortion of an event's duration that occurs when the event

243 is inserted in a series. It constitutes one of the oldest and least understood phenomena of subjective

244 time perception. ${ }^{16,17,18}$ A set of models have been proposed to try and explain how TOEs are generated.

245 In the sensation-weighting model, ${ }^{8}$ temporal distortions would arise because the sensory effects

246 produced by $\mathrm{S}$ and $\mathrm{C}$ are differentially weighted before they are discriminated. In the difference

247 model, ${ }^{6}$ TOEs depend on two components: 1) Desensitization caused by short inter-stimulus intervals

248 (ISIs) — akin to a form of attentional blink ${ }^{19}$ — not allowing the sensory system to reset back to its

249 initial state; ${ }^{5}$ ) an idiosyncratic response bias in picking a specific stimulus (always picking the first, or

250 the second one). Both models predict that by improving stimulus encoding processes, such as by

251 increasing ISI and thus temporal attention to the second stimulus in a series, TOE should decrease.

252 We verified this stance in our previous work ${ }^{4}$ using a visual two-interval forced-choice (2IFC) task

253 with empty events in a fixed order, with the comparison (C) event always following the standard (S)

254 event. However, all accounts missed the crucial point of explaining how TOEs are generated under

255 uncertain sensory evidence and limited attention resources.

256 Although a meta-analysis ${ }^{21}$ suggested that temporal sensitivity decreases when the Standard 257 event is displayed in the $2^{\text {nd }}$ position, our results demonstrate no effect of $S$ position on temporal 258 sensitivity, which was modulated only by the ISI factor. We conclude that TOEs are not elicited by 259 differences in sensory precision. Our analysis of key presses shows that participants were not biased 260 in hitting the key for " $\mathrm{S}$ " or " $\mathrm{C}$ ". That is, TOEs are not generated by an absolute positional bias, as 261 predicted by the difference model. ${ }^{6}$

262 Since TOEs occur during serial discrimination tasks, we tested whether event duration 263 dynamics is the primary source of distortions by flipping the positions of standard (S) and comparison

264 (C) events in two separate behavioral experiments. First, we replicated the finding that increasing the 265 temporal interval between first and second event minimizes TOEs for $\mathrm{S}$ in both $1^{\text {st }}$ and $2^{\text {nd }}$ position, 266 although significantly less frequently for $S$ in $2^{\text {nd }}$ position. These results can be explained by 267 the beneficial effects of allocating attention in time, more oriented to the encoding unpredictable 268 events $(\mathrm{C}) .^{7,20,15}$

Second, a Bayesian modelling of accuracy at each sensory level $(\Delta 100, \Delta 60,20 \Delta)$ showed 270 that, while most TOEs occur for short ISIs and small to medium sensory evidence, they tend to cluster 
271 in a specific serial condition: When the first stimulus in a series is shorter than the second stimulus,

272 regardless of whether it is S or C. In such a case, participants were biased to say that the first event

273 was longer, consistently making mistakes.

274 When the first stimulus was truly larger than the second, performance was significantly above

275 chance, suggesting optimal processing even under uncertain sensory circumstances (small and medium

276 sensory evidence, 20/60 ms). This prefigures a novel order bias in serial perception based on duration-

277 dependent relative positions of stimuli, which can only be partially counteracted by increasing

278 temporal attention. Attention helps when $\mathrm{S}$ is in the first position, as it enhances the encoding of the

279 C stimulus whose duration is unpredictable.

280 Our new model finally explains that time order errors arise under sensory uncertainty because

281 of serial perceptual encoding inefficiency. Future research is needed to uncover the physiological basis

282 of such a strong expectation about the temporal statistics of incoming stimuli.

\section{STAR Methods}

\section{Participants}

286 Experiment 1. Part of the results for experiment 1 were previously published. ${ }^{4}$ This dataset has a

287 sample of 52 participants (34 female; ages: 18-33; mean age: 24.42). We removed participants with an

288 accuracy $\leq 55 \%$, as this is an indicator that they completed the task by chance. One participant was

289 removed due to his low accuracy. For each participant we computed the goodness-of-fit of the

290 psychometric function $\left(R^{2}\right)$. Participants with a $R^{2}$ value lower than two standard deviations away

291 from the mean were removed. Seven participants were removed from analysis following this

292 procedure. For the dependent variables WF and CE, we also discarded extreme outliers. We identified

293 these outliers by using two standard deviations. Two participants were discarded for being marked as

294 extreme outlier in experiment 1 . Therefore, the final sample included the data from 42 participants (26

295 female; ages: 18-33; mean: 24.14).

Experiment 2. We had an initial sample of 58 participants in experiment 2 (45 female; ages:

297 18-37; mean age: 25.41). We applied the same procedure as in experiment 1 for discarding 298 outliers. Four participants were removed from analysis due to their low performance of correct 
responses $(<55 \%)$. Nine participants were removed from analysis due to their low $R^{2}$ values. One

300 participant was discarded for being marked as extreme outliers in the WF. Therefore, the final analysis

301 included the data from 44 participants (34 female; ages: 18-32; mean: 25.22). In total, we report on the

302 behavior of 86 participants.

303 Individuals were recruited through online advertisements. Participants self-reported normal

304 or corrected vision and had no history of neurological disorders. Up to three participants were tested

305 simultaneously at computer workstations with identical configurations. They received 10 euros per

306 hour for their participation. The studies were approved by the Ethics Committee of the Max Planck

307 Society. Written informed consent was obtained from all participants previous to the experiment.

309 Design

310 We used a classical interval discrimination task by using a 2IFC design, where participants were

311 presented with two visual durations: $\mathrm{S}$ and $\mathrm{C} \cdot{ }^{22,23} \mathrm{~S}$ had a magnitude of $120 \mathrm{~ms}$ and was always displayed

312 in the first position in experiment 1, but in the second position in experiment 2. In both experiments,

313 we used three magnitudes for the step comparisons $\Delta$ between S and C: 20, 60, and $100 \mathrm{~ms}$. We

314 derived the magnitudes for the $\mathrm{C}$ stimuli as $S \pm \Delta$, which resulted in the next C durations: 20, 60, 100,

315 140, 180, and $220 \mathrm{~ms}$. For the ISI, we used four durations: 400, 800, 1600, and 2000 ms. For each

316 trial, the inter-trial interval (ITI) was randomly chosen from a uniform distribution between 1 and 3

317 seconds. Participants judged whether the $\mathrm{S}$ or $\mathrm{C}$ interval was the longer duration, and responded by

318 pressing one of two buttons on an RB-740 Cedrus Response Pad (cedrus.com, response time jitter $<1$

319 ms, measured with an oscilloscope).

\section{Stimuli and Apparatus}

322 We used empty visual stimuli, which were determined as a succession of two blue disks with a diameter

323 of $1.5^{\circ}$ presented on a gray screen. ${ }^{24}$ We used empty stimuli to ensure that participants were focused

324 on the temporal properties of the stimuli. ${ }^{25}$ All stimuli were created in MATLAB R2018b

325 (mathworks.com), using the Psychophysics Toolbox extensions. ${ }^{26,27,28}$ Stimuli were displayed on an

326 ASUS monitor (model: VG248QE; resolution: 1,920 x 1,080; refresh rate: $144 \mathrm{~Hz}$; size: 24 in) at a

327 viewing distance of $60 \mathrm{~cm}$. 


\section{Protocol (Task)}

330 The experiment was run in a single session of 70 minutes. Participants completed a practice set of

331 four blocks (18 trials in each block). All sessions consisted of the presentation of one block for each

332 ISI condition. Each block was composed of 120 trials and presented in random order. In order to

333 avoid fatigue, participants always had a break after 60 trials. Each trial began with a black fixation cross

334 (diameter: $0.1^{\circ}$ ) displayed in the center of a gray screen. Its duration was randomly selected from a

335 distribution between 400 and $800 \mathrm{~ms}$. After a blank interval of $500 \mathrm{~ms}$, S was displayed and followed

336 by $\mathrm{C}$, after one of the ISI durations.

337 Participants were instructed to compare the durations of the two stimuli by pressing the key

338 "left", if S was perceived to have lasted longer, and the key "right" if C was perceived to have lasted

339 longer. After responding, they were provided with immediate feedback: the fixation cross color

340 changed to green when the response was correct, and to red when the response was incorrect.

\section{Data analysis}

343 The data analysis was implemented with Python 3.7 (python.org) using the ecosystem SciPy (scipy.org)

344 and the libraries Pandas, ${ }^{29}$ Seaborn, ${ }^{30}$ and Pingouin. ${ }^{31}$ Frequentist statistical analyses were executed in

345 Pingouin. Bayesian statistical analyses were implemented using the BayesFactor package for R. ' All

346 data and statistical analyses were performed in Jupyter Lab (jupyter.org).

347 To endorse open science practices and transparency on statistical analyses, we used

348 JASP $^{32}$ (jasp-stats.org) for providing statistical results (data, plots, distributions, tables and post hoc

349 analyses) of both Frequentist and Bayesian analyses in a graphical user-friendly interface. These results

350 can be consulted at Open Science Framework as annotated .jasp files (osf.io/jkzq4/). As JASP uses

351 the BayesFactor package as a backend engine, the default prior distributions of this package were the

352 same for JASP. Modelling of the Bayesian rate-comparison model ${ }^{14}$ was done in MATLAB R2018b

353 (mathworks.com) using JAGS 4.3.0 (w3w.mcmc-jags.sourceforge.net/). 


\section{Psychometric curves}

356 Responses were modeled using a 6-point psychometric function using the nonlinear least-squares fit

357 in Python. We plotted the six $\mathrm{C}$ durations on the $x$-axis and the probability of responding " $\mathrm{C}$ longer

358 than $\mathrm{S}$ " on the $y$-axis. We parameterized psychometric functions by using the distribution of a logistic

359 function $f$, which is given by

$$
f(x)=\frac{L}{1+\exp ^{-(x-\mu) / \beta}}
$$

362 where $L$ is the maximum value of $f, x$ is the magnitude of the $C$ stimuli, $\mu$ is the $x$-value at the mid363 point of the psychometric function, and $\beta$ parametrizes the slope of the logistic function. Two indices 364 of temporal performance are extracted from this fitting: a marker for the perceived event duration, 365 and a marker for the sensory temporal precision. ${ }^{34}$ The perceived duration was measured via the PSE 366 ( $\mu$ in equation 1), i.e., the value on the $x$-axis that corresponds to the $50 \%$ value on the $y$-axis. ${ }^{34} \mathrm{We}$ 367 derived the CE from the PSE, which is defined as the difference between the PSE and the magnitude 368 of the physical duration $\phi_{s}$ of S:

$$
C E=\mu-\phi_{s}
$$

372

373 Positive CEs indicate that the $\mathrm{C}$ stimuli were perceived as shorter than the $\mathrm{S}$ stimulus. The temporal

374 sensitivity was measured by using the JND, which is defined as being half the interquartile range of

375 the fitted function $\left(J N D=\frac{x_{.75}-x_{.25}}{2}\right)$, where $x_{.75}$ and $x_{.25}$ denote the point values on the $y$-axis that 376 output $25 \%$ and $75 \%$ "longer" responses. The smaller the JND, the higher the discrimination 377 sensitivity of the sensory system. The JND is obtained from the slope ( $\beta$ in equation 1) of the fitted 378 function:

$$
J N D=\beta \cdot \log \left(\frac{0.75}{0.25}\right)
$$




\section{Statistical analyses}

383 Frequentist analyses. Frequentist statistical analyses are available at Open Science Framework 384 (osf.io/jkzq4/). For the Frequentist analyses the level of statistical significance to reject the null

385 hypothesis $H_{0}$ was set to $\alpha=0.05$. To test for significant changes in the dependent variables, we 386 implemented a repeated measures ANOVA across ISI levels in both experiments. We carried out post 387 hoc comparisons by using the Bonferroni correction $\left(\frac{\alpha}{4}\right)$.

Bayesian Model Comparison. To contrast results from both experiments, we applied a Bayes Factor approach to ANOVA by using Bayesian Model Comparison (BMC). ${ }^{13,35}$ Tod do that, we implemented Bayes' rule for obtaining the posterior distribution $p(\theta \mid D)$, where $D$ expresses the observed data, under the model specification $M_{1},{ }^{11,14}$ which is given by

$$
p\left(\theta \mid D, M_{1}\right)=\frac{p\left(D \mid \theta, M_{1}\right) p\left(\theta \mid M_{1}\right)}{p\left(D \mid M_{1}\right)}
$$

where $p\left(D \mid \theta, M_{1}\right)$ denotes the likelihood, $p=\left(\theta \mid M_{1}\right)$ expresses the prior distribution, and the marginal likelihood is expressed by $p\left(D \mid M_{1}\right)$. Bayes factor ANOVA compares the predictive performance of competing models. Thus, for evaluating the relative probability of the data $D$ under competing models we computed the Bayes factor $(\mathrm{BF})$ : Let $\mathrm{BF}_{10}$ express the Bayes factor between a null model $M_{0}$ versus an alternative model $M_{1}$. The predictive performance of these models is given

401 by the probability ratio obtained by dividing the marginal likelihoods of $M_{1}$ and $M_{0}$ :

$$
B F_{10}=\frac{p\left(D \mid M_{1}\right)}{p\left(D \mid M_{0}\right)}
$$

406 In this case, $\mathrm{BF}_{10}$ expresses to which extent the data support the model $M_{1}$ over $M_{0}$, whereas $\mathrm{BF}_{01}$

407 indicates the Bayes factor in favor $M_{0}$ over $M_{1}$. BF values $<0$ give support to $M_{0}$, whereas $\mathrm{BF}>1$ 
analysis of the dependent variables we build four alternative models ( $M_{\mathrm{E}}, M_{\mathrm{ISI}}, M_{\mathrm{ISI}+\mathrm{E}}$, and the $\left.M_{\mathrm{Int}}\right)$ for

410 quantifying the effect of two factors: ISI and Standard position. The $M_{\mathrm{E}}$ model uses the Standard

411 position as predictor, whereas the $M_{\text {ISI }}$ model uses the ISI. The $M_{\text {ISI+E }}$ model uses both factors as

412 predictors (the ISI and the Standard position), and the $M_{\text {Int }}$ model uses again both factors but includes

413 also their interaction (ISI * Experiment). The prior model probability $p(\mathrm{M})$ of each model was set to

414 be equal, i.e., prior model odds of 0.2. We compare each model to the null model $M_{0}$ and provided the

$415 \mathrm{BF}$. The model with the highest BF was selected as the best model, and thus is the inferential model

416 that explain the data more accurately.

417 Bayesian post hoc testing is based on pairwise comparisons using Bayesian t-tests with a

418 Cauchy $\left(0, r=\frac{1}{\sqrt{2}}\right)$ prior. For post hoc testing, the posterior odds are corrected for multiple testing

419 by fixing to 0.5 the prior probability that the null hypothesis holds across comparisons. ${ }^{36}$ For all our

420 analyses we used the default prior values for Bayes factor ANOVA, ${ }^{37,38}$ which are also the default

421 values in the BayesFactor package and JASP.

422 Bayesian rate-comparison model. To test individual differences on the accuracy-rate $\theta$

423 between the conditions a ("First stimulus longer") and b (“Second stimulus longer"), we used the

424 Bayesian comparison-rate model (see Fig. 4a) for computing differences between $\theta^{a}$ and $\theta^{b} \cdot{ }^{14} \mathrm{We}$

425 deployed the Bayesian comparison-rate model for within subject comparisons at each ISI level and

426 modeled differences between conditions as having Gaussian distributions. That is, with mean $\mu$ and

427 standard deviation $\sigma$ :

$\mu \sim \operatorname{Gaussian}(0,1)$

$\sigma \sim \operatorname{Uniform}(0,10)$

431 This model receives two inputs for each subject $i$ : the number of trials $n$ and the total number of 432 correct responses $s$. We assumed that the rate parameter $\theta$ follows a binomial distribution. In order 433 to model $\theta$ as a normally distributed variable, we applied a probit transformation, which transforms $\theta$ 434 (a real number) into a probability $\phi:^{39}$ 


$$
\begin{gathered}
s_{i}^{\mathrm{b}} \sim \operatorname{Binomial}\left(\theta_{i}^{\mathrm{b}}, n^{\mathrm{b}}\right) \\
\theta_{i}^{\mathrm{a}} \leftarrow \Phi\left(\phi_{i}^{\mathrm{a}}\right) \\
\theta_{i}^{\mathrm{b}} \leftarrow \Phi\left(\phi_{i}^{\mathrm{b}}\right)
\end{gathered}
$$

441

442 We added a variable $\delta$ to quantify the strength of the effect size:

$$
\delta \sim \operatorname{Gaussian}(0,1)
$$

445 Then, we modeled the effect $\alpha$ as random effect. That is, with a Gaussian distribution of mean $\mu_{\alpha}$ 446 and standard deviation $\sigma_{\alpha}$ :

$$
\mu_{\alpha} \leftarrow \delta \sigma_{\alpha}
$$

$$
\sigma_{\alpha} \text { Uniform }(0,10)
$$

$$
\alpha_{i} \sim \operatorname{Gaussian}\left(\mu_{\alpha}, \frac{1}{\sigma}\right)
$$

450 Thus, differences between conditions are given by adding the effect $\alpha$ to one of the transformed 451 rates $\phi$ :

$$
\phi_{i}^{\mathrm{b}} \sim \operatorname{Gaussian}\left(\mu, \frac{1}{\sigma^{2}}\right)
$$

456 To run this model, we generated 50 chains with 100000 samples, which generated a total of 5000000 457 samples. We discarded the first 1000 burn-in samples. Posterior distributions of this model show 458 visually the strength of the effect size $\delta$. We computed the Bayes factors for this model using the 459 Savage-Dickey method. ${ }^{40,14}$ 


\section{Acknowledgements}

462 We thank Lauren Fink and Luigi Acerbi for critical suggestions.

\section{Author contributions}

464 Research question: FS and AT. Study design: All authors. Testing and data collection: FS. Data analysis 465 and writing original draft: FS and AT. Writing, review \& editing: all authors.

\section{Declaration of interests}

467 The authors declare no competing interests.

468

\section{Supplemental information}

470 Strong sensory evidence $( \pm \Delta 100)$. Results of experiment 1 yielded anecdotal evidence in favor

471 of $H_{0}$ in all conditions: $\mathrm{ISI}_{400}, \mathrm{ISI}_{800}, \mathrm{ISI}_{1600}$ and $\mathrm{ISI}_{2000}\left(\mathrm{BF}_{10}=0.29 ; \mathrm{BF}_{10}=0.51 ; \mathrm{BF}_{10}=0.81, \mathrm{BF}_{10}=\right.$

472 0.94; respectively; Fig SI 1a). We found similar results in experiment 2 for the conditions

$473 \mathrm{ISI}_{400,} \mathrm{ISI}_{800}, \mathrm{ISI}_{1600}$ and $\mathrm{ISI}_{2000}\left(\mathrm{BF}_{10}=0.38 ; \mathrm{BF}_{10}=0,58 ; \mathrm{BF}_{10}=0.52 ; \mathrm{BF}_{10}=0.34\right.$; respectively; Fig

474 SI 1b). That is, when sensory evidence for $\mathrm{C}$ is strong, no duration-dependent bias can be detected.

475

476

477 
a)

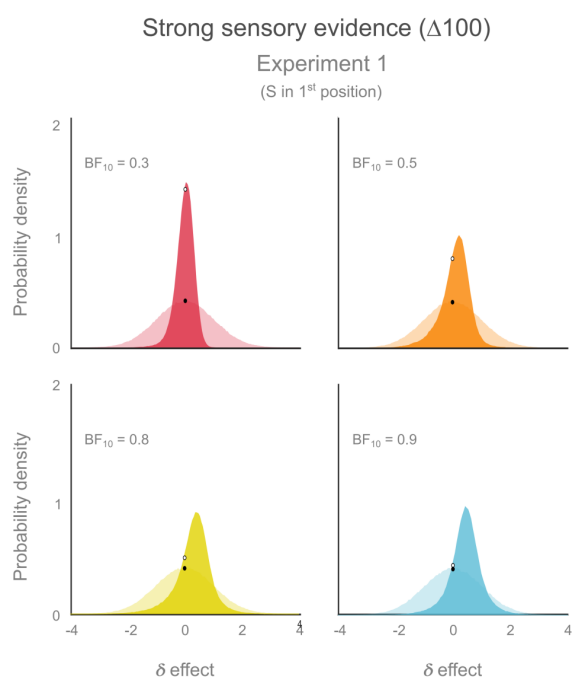

b)

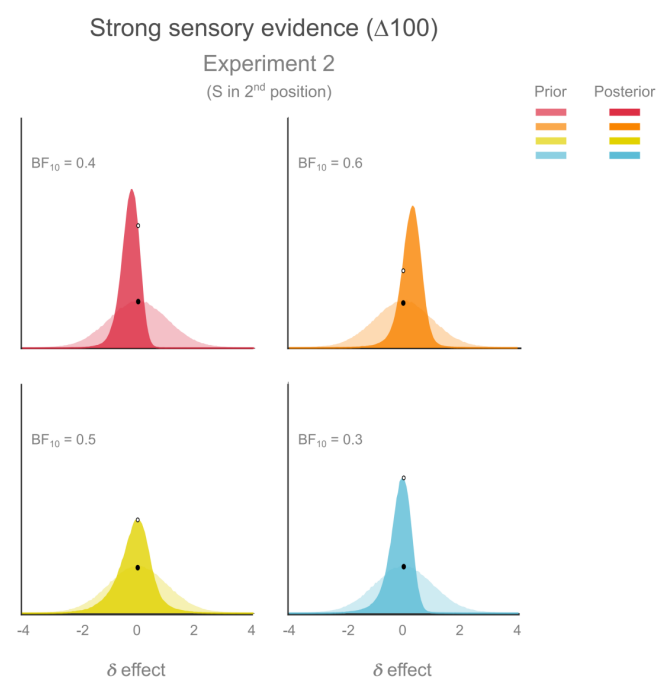

479 Fig SI 1. Strong sensory evidence category and posteriors distributions of the Bayesian rate480 comparison model. a) Results yielded that when the Standard duration is in the first position 481 (Experiment 1), at each ISI level there is no evidence for $H_{1}$. Thus, no differences between the 482 conditions "First stimulus longer" and "Second stimulus longer" can be claimed. This can be visually 483 verified by the overlapping distributions at each ISI level. b) The same was true when the Standard 484 event was displayed in the second position (Experiment 2): The Bayes factor showed no evidence 485 for $H_{1}$, but anecdotal evidence for $H_{0}$. Thus, when there is a strong sensory difference between stimuli, 486 no differences in accuracy rate between the conditions "Long stimulus first" and "Long stimulus 487 second" can be claimed. 


\section{a)}

\section{Strong sensory evidence $(\Delta 20)$}
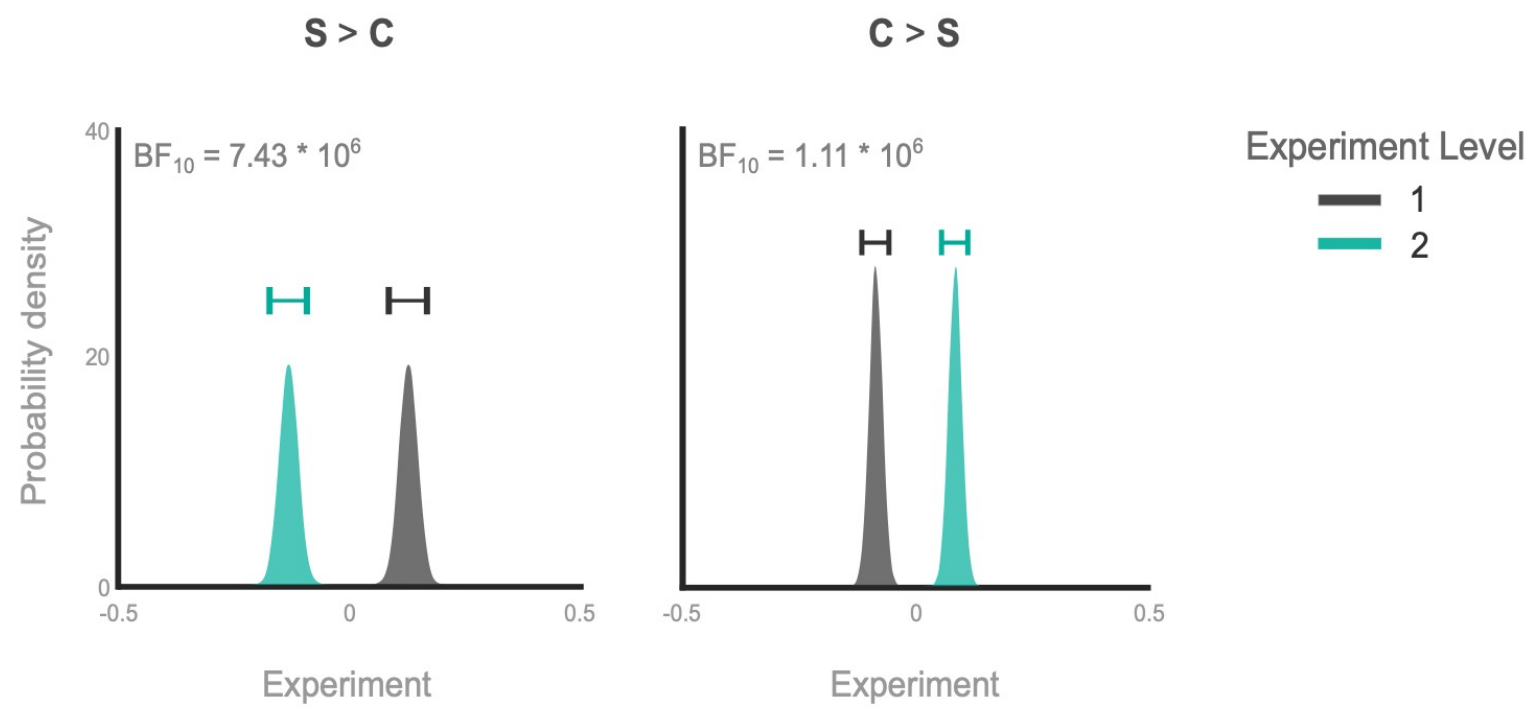

490 Fig SI 2. Results of the Bayesian Binomial analyses of the Strong sensory evidence condition. Bayes 491 factors $\left(\mathrm{BF}_{10}\right)$ revealed that there is no individual bias in responses either in experiment 1 or 492 experiment 2. Results yielded evidence in favor of the null hypothesis (no differences between 493 responses "First stimulus longer" or "Second stimulus longer"). 


\section{References}

502 1.Jazayeri, M. \& Shadlen, M. N. A Neural Mechanism for Sensing and Reproducing a Time Interval.

503 Current Biology 25, 2599-609 (2015).

504 2.Nakajima, Y., ten, H. G., Hilkhuysen, G. \& Sasaki, T. Time-shrinking: a discontinuity in the

505 perception of auditory temporal patterns. Percept Psychophys 51, 504-7 (1992).

506 3.Allan, L. G. \& Gibbon, J. A new temporal illusion or the TOE once again? Perception \& Psychophysics

507 55, 227-229 (1994).

508 4.Sierra, F., Poeppel, D. \& Tavano, A. How to minimize time distortions. (2020)

509 doi:10.31234/osf.io/2w9ev.

510 5.Buonomano, D. V., Bramen, J. \& Khodadadifar, M. Influence of the interstimulus interval on

511 temporal processing and learning: testing the state-dependent network model. Philos Trans R Soc Lond

512 B Biol Sci 364, 1865-73 (2009).

513 6.Alcalá-Quintana, R. \& García-Pérez, M. A. A model for the time-order error in contrast

514 discrimination. Quarterly Journal of Experimental Psychology 64, 1221-1248 (2011).

515 7.Nobre, A. C., Correa, A. \& Coull, J. T. The hazards of time. Current Opinion in Neurobiology 17, 465-

516470 (2007).

517 8.Hellström, . A. Factors producing and factors not producing time errors: An experiment with

518 loudness comparisons. Perception \& Psychophysics 23, 433-444 (1978).

519 9.Morey, R. D. \& Rouder, J. N. BayesFactor: Computation of Bayes Factors for Common Designs.

520 (2018).

521 10.Lapid, E., Ulrich, R. \& Rammsayer, T. On estimating the difference limen in duration

522 discrimination tasks: A comparison of the 2AFC and the reminder task. Perception \& Psychophysics 70,

523 291-305 (2008).

524 11.Jeffreys, H. The theory of probability. (Oxford University Press, 1961).

525 12.Wagenmakers, E.-J. A practical solution to the pervasive problems ofp values. Psychonomic Bulletin

526 \& Review 14, 779-804 (2007).

527 13.Rouder, J. N., Engelhardt, C. R., McCabe, S. \& Morey, R. D. Model comparison in ANOVA.

528 Psychonomic Bulletin \& Review 23, 1779-1786 (2016).

529 14.Lee, M. D. \& Wagenmakers, E.-J. Bayesian Cognitive Modeling. (Cambridge University Press, 2013). 
530 15.Cravo, A. M., Rohenkohl, G., Wyart, V. \& Nobre, A. C. Temporal Expectation Enhances Contrast

531 Sensitivity by Phase Entrainment of Low-Frequency Oscillations in Visual Cortex. Journal of

532 Neuroscience 33, 4002-4010 (2013).

533 16.Fechner, G. Elements of Pshycophysics. (Holt., 1860/1966).

534 17.Allan, L. G. The perception of time. Perception \& Psychophysics 26, 340-354 (1979).

535 18.Hellström, . Time errors and differential sensation weighting. Journal of Experimental Psychology:

536 Human Perception and Performance 5, 460-477 (1979).

537 19.Marois, R., Chun, M. M. \& Gore, J. C. Neural Correlates of the Attentional Blink. Neuron 28, 299-

$538308(2000)$.

539 20.Rohenkohl, G., Cravo, A. M., Wyart, V. \& Nobre, A. C. Temporal Expectation Improves the

540 Quality of Sensory Information. Journal of Neuroscience 32, 8424-8428 (2012).

541 21.Dyjas, O., Bausenhart, K. M. \& Ulrich, R. Trial-by-trial updating of an internal reference in

542 discrimination tasks: evidence from effects of stimulus order and trial sequence. Atten Percept Psychophys

543 74, 1819-41 (2012).

544 22.Wright, B. A., Buonomano, D. V., Mahncke, H. W. \& Merzenich, M. M. Learning and

545 Generalization of Auditory TemporalInterval Discrimination in Humans. The Journal of Neuroscience 17,

546 3956-3963 (1997).

547 23.Mauk, M. D. \& Buonomano, D. V. The neural basis of temporal processing. Annual Review of

$548 \quad$ Neuroscience 27, 307-340 (2004).

549 24.Kliegl, K. M. \& Huckauf, A. Perceived duration decreases with increasing eccentricity. Acta

550 Psychologica 150, 136-145 (2014).

551 25.Grondin, S., Meilleur-Wells, G., Ouellette, C. \& Macar, F. Sensory effects on judgments of short

552 time-intervals. Psychological Research 61, 261-8 (1998).

553 26.Brainard, D. H. The Psychophysics Toolbox. Spatial Vision 10, 433-436 (1997).

554 27.Pelli, D. G. The VideoToolbox software for visual psychophysics: transforming numbers into 555 movies. Spatial Vision 10, 437-442 (1997).

556 28.Kleiner, M. et al. What's new in psychtoolbox-3. Perception 36, 1-16 (2007).

557 29.The pandas development team. pandas-dev/pandas: Pandas. (2020) doi:10.5281/zenodo.3509134.

558 30.Waskom, M. L. seaborn: statistical data visualization. Journal of Open Source Software 6, 3021 (2021).

559 31.Vallat, R. Pingouin: statistics in Python. Journal of Open Source Software 3, 1026 (2018).

560 32.JASP Team. JASP (Version 0.14.1)[Computer software]. (2020). 
561 33.Plummer, M. \& others. JAGS: A program for analysis of Bayesian graphical models using Gibbs

562 sampling. in Proceedings of the 3rd international workshop on distributed statistical computing vol. 124 1-10

563 (2003).

564 34.Grondin, S. Discriminating time intervals presented in sequences marked by visual signals. Perception

565 \& Psychophysics 63, 1214-1228 (2001).

566 35.Rouder, J. N., Morey, R. D., Verhagen, J., Swagman, A. R. \& Wagenmakers, E. J. Bayesian analysis

567 of factorial designs. Psychol Methods 22, 304-321 (2017).

568 36.Doorn, J. van et al. The JASP guidelines for conducting and reporting a Bayesian analysis.

569 Psychonomic Bulletin \& Review (2020).

570 37.Wagenmakers, E. J. et al. Bayesian inference for psychology. Part I: Theoretical advantages and

571 practical ramifications. Psychonomic Bulletin \& Review 25, 35-57 (2018).

572 38.Rouder, J. N., Morey, R. D., Speckman, P. L. \& Province, J. M. Default Bayes factors for ANOVA

573 designs. Journal of Mathematical Psychology 56, 356-374 (2012).

574 39.Annis, J., Dubé, C. \& Malmberg, K. J. A Bayesian approach to discriminating between biased

575 responding and sequential dependencies in binary choice data. Decision 5, 16-41 (2018).

576 40.Dickey, J. M. The Weighted Likelihood Ratio Linear Hypotheses on Normal Location Parameters.

577 The Annals of Mathematical Statistics 42, 204-223 (1971).

578 41.Hellström, . A. \& Rammsayer, T. Time-order errors and standard-position effects in duration

579 discrimination: An experimental study and an analysis by the sensation-weighting model. Attention

580 Perception, \& Psychophysics 77, 2409-2423 (2015).

581 42.Rouder, J. N., Engelhardt, C. R., McCabe, S. \& Morey, R. D. Model comparison in ANOVA.

582 Psychonomic Bulletin \& Review 23, 1779-1786 (2016).

583 43.Nobre, A. C. Orienting attention to instants in time. Neuropsychologia 39, 1317-1328 (2001). 